Publisher: Elsevier Science BV, PO Box 211, 1000 AE Amsterdam, Netherlands

Subject category: Chemistry, Analytical, Forensic Science

ISSN: 0379-0738

DOI: doi:10.1016/j.forsciint.2010.03.019

http://www.sciencedirect.com/science?_ob=ArticleURL\&_udi=B6T6W-4YXOBRD-

$2 \&$ user $=2347467 \&$ ccoverDate $=06 \% 2 \bar{F} 15 \% 2 F 2010 \&$ rdoc $=1 \&$ fmt=high\&_orig=search\&_origin

$=$ search\&_sort $=d \& \_$docanchor $=\&$ view $=c \&$ acct $=$ C000057013\&_version $=1 \&$ _urlVersion $=0$ \&_useri $\mathrm{d}=234746 \overline{7} \& \mathrm{md} 5=\overline{4} 7637 \mathrm{e} 7 \mathrm{c} 4 \mathrm{cf} 21 \mathrm{~b} 89 \mathrm{a94} \overline{7} \mathrm{dad} 7 \mathrm{~d} 9 \mathrm{ae} 1 \mathrm{a} 98 \&$ searchtype $=\mathrm{a}$

\title{
VARIATION IN AMINO ACID AND LIPID COMPOSITION OF LATENT FINGERPRINTS
}

\author{
Ruth S Croxton ${ }^{\mathrm{a},{ }^{*}, \text { Mark G Baron }^{\mathrm{a}} \text {, David Butler }}{ }^{\mathrm{b}}$, Terry Kent ${ }^{\mathrm{c}}$, Vaughn G Sears ${ }^{\mathrm{c}}$. \\ ${ }^{a}$ School of Natural and Applied Sciences, University of Lincoln, Brayford Pool, Lincoln, LN6 7TS, \\ $U K$. \\ ${ }^{b}$ School of Science and Technology, Nottingham Trent University, Clifton Campus, Nottingham, \\ NG11 8NS, UK.
}

${ }^{c}$ Home Office Scientific Development Branch, Sandridge, St Albans, AL4 9HQ, UK.

*Corresponding author:

Dr. R. Croxton

Postal address: School of Natural and Applied Sciences, University of Lincoln, Brayford Pool, Lincoln,

LN6 7TS, UK.

Email: rcroxton@lincoln.ac.uk

Telephone: +44 (0)1522 886789

Fax: +44 (0)1522 886791 


\title{
VARIATION IN AMINO ACID AND LIPID COMPOSITION OF LATENT FINGERPRINTS
}

\author{
Abstract \\ The enhancement of latent fingerprints, both at the crime scene and in the laboratory using an array of \\ chemical, physical and optical techniques, permits their use for identification. Despite the plethora of \\ techniques available, there are occasions when latent fingerprints are not successfully enhanced. An \\ understanding of latent fingerprint chemistry and behaviour will aid the improvement of current techniques \\ and the development of novel ones. In this study the amino acid and fatty acid content of 'real' latent \\ fingerprints collected on a non-porous surface was analysed by gas chromatography-mass spectrometry. \\ Squalene was also quantified in addition. Hexadecanoic acid, octadecanoic acid and cis-9-octadecenoic \\ acid were the most abundant fatty acids in all samples. There was, however, wide variation in the relative \\ amounts of each fatty acid in each sample. It was clearly demonstrated that touching sebum-rich areas of \\ the face immediately prior to fingerprint deposition resulted in a significant increase in the amount of fatty \\ acids and squalene deposited in the resulting 'groomed' fingerprints. Serine was the most abundant amino \\ acid identified followed by glycine, alanine and aspartic acid. The significant quantitative differences \\ between the 'natural' and 'groomed' fingerprint samples seen for fatty acids were not observed in the case \\ of the amino acids. This study demonstrates the variation in latent fingerprint composition between \\ individuals and the impact of the sampling protocol on the quantitative analysis of fingerprints.
}

\section{Key words}

latent fingerprint, gas chromatography-mass spectrometry, ethyl chloroformate, amino acid, fatty acid, squalene 


\section{Introduction}

Fingerprints have a key role in crime detection. The comparison of fingerprints recovered from a crime scene with either fingerprints taken from a suspect or with an individual's record held on a database provides a powerful tool. Fingerprint evidence found at a crime scene may be described as visible, plastic (impression) or latent. Latent fingerprints are the most commonly encountered type at crime scenes and are not readily visible. Visualisation techniques are a commonplace tool employed to enhance such fingerprints, enabling them to be recorded and identified. A wide array of chemical, physical and optical techniques is used including ninhydrin, cyanoacrylate (superglue) fuming and powdering [1]. Improvements of current techniques are continually sought providing greater sensitivity and wider applications.

The identification of novel enhancement techniques occupies much of the research in this field. A variety of strategies are employed in the development of new latent fingerprint enhancement techniques such as the exploitation of chemical analogues of existing reagents or the application of reagents used in other fields [2,3]. A further strategy involves the targeting of specific compounds in latent fingerprint residue, for example the detection of drug metabolites using antibodies immobilised on nanoparticles or the immunodetection of peptides and proteins $[4,5,6]$. In some instances these enhancement techniques may provide information about personal habits, as well as develop ridge detail [4,5]. Novel applications of analytical techniques, including micro-X-ray fluorescence, Fourier transform infrared spectroscopy and time-of-flight secondary ion mass spectrometry are also being used in the visualisation of latent fingerprints and to study their behaviour $[7,8,9,10]$. Prior knowledge of the chemical composition of latent fingerprint residue, and its behaviour after deposition on a surface, would aid the identification of target components, and in particular those which are common to all fingerprints, to allow for a universal reagent or technique to be developed.

Latent fingerprints are composed of the natural secretions of glands in the skin, principally eccrine and sebaceous glands. Eccrine sweat consists predominantly of water and the remainder is a highly complex mixture of organic (e.g. amino acids, proteins and lactate) and inorganic material (e.g. $\mathrm{Na}^{+}, \mathrm{K}^{+}, \mathrm{Cl}^{-}$and 
trace metal ions). Sebaceous secretions are predominantly composed of fatty acids, glycerides, cholesterol, squalene and a variety of lipid esters. Environmental contaminants, e.g. bacteria spores, dust, cosmetics, hair products and tobacco products, may also contribute [11]. Whilst extensive data are available on the constituents of sweat, relatively little data is currently available on the specific composition of 'real' latent fingerprints and how it changes with time after deposition.

Many of the latent fingerprint studies conducted to date have studied the lipid components namely fatty acids, long chain fatty acid esters, squalene, cholesterol and wax esters. The lipid composition of latent fingerprints has been found to vary between individuals with age and gender $[12,13,14]$. Chemical changes that occur following deposition and environmental conditions are an important factor [15]. Unsaturated compounds such as squalene, oleic (cis-9-octadecenoic) and palmitoleic (cis-9-hexadecenoic) acids are predominantly lost over time, whilst saturated compounds essentially remain unchanged [13,15]. Lower molecular weight compounds, such as short chain fatty acids appear in the fingerprint as it ages and are formed as unsaturated compound degradation products. Degradation of squalene is more rapid in light conditions compared to dark [15]. Mountfort et al. [16] identified squalene oxidation products in aged latent fingerprints including squalene monohydroperoxide and squalene epoxide and suggested that these and other oxidation products could be potential targets for novel visualisation reagents.

It is estimated that the average amino acid content of a fingerprint is $250 \mathrm{ng}$ [17]. Amino acids present on the surfaces of the hands have been studied previously in the 1960s, although not in the context of latent fingerprints per se $[18,19,20]$. The relative concentrations of free amino acids are comparable between the studies with serine, glycine, ornithine and alanine being most abundant. Cuthbertson [21] studied the aqueous components of latent fingerprints including amino acids and chloride. Chloride concentration was found to vary between donors (age, occupation) and was affected by digit used and the porosity of the substrate. The mean concentration of chloride was almost three times greater in those fingerprints deposited on filter paper compared to those on aluminium foil. 
A novel procedure which permits the simultaneous analysis of lipid and nitrogenous material, namely fatty acids, squalene and amino acids, in 'real' latent fingerprints has previously been reported [22]. The protocol aimed to allow the analysis of the natural distribution of a variety of substances in latent fingerprints. One of the key objectives of the protocol was to ensure that the fingerprint collection protocol mimics as closely as possible fingerprints as would be found at a crime scene. The use of this protocol to study the qualitative and quantitative variation between individuals in the amino acid and fatty acid content of their latent fingerprints is reported here. The impact of the sampling protocol on fingerprint residue composition is also explored to inform our interpretation of fingerprint studies.

\section{Materials and Methods}

\section{Materials}

L-asparagine (Asn), L-p-chlorophenylalanine (cPhe), L-cysteine (Cys), 4-hydroxy-L-proline (HPro), Lornithine hydrochloride (Orn) and L-tryptophan (Trp) (all > 99\%), octanoic (C8), decanoic (C10), undecanoic (C11), dodecanoic (C12), tridecanoic (C13), tetradecanoic (C14), cis-9-tetradecenoic (C14:1), pentadecanoic (C15), hexadecanoic (C16), cis-9-hexadecenoic (C16:1), octadecanoic (C18), cis-9octadecenoic (C18:1), cis-9,12-octadecadienoic (C18:2) and eicosanoic (C20) acids (all $\geq 98 \%)$, pyridine (ACS reagent) and squalene (98\%) were obtained from Sigma-Aldrich (Gillingham, Dorset). The amino acid standard AA-S-18, containing L-alanine (Ala), L-arginine (Arg), L-aspartic acid (Asp), glycine (Gly), L-glutamic acid (Glu), L-isoleucine (Ile), L-leucine (Leu), L-lysine (Lys), L-methionine (Met), Lphenylalanine (Phe), L-proline (Pro), L-serine (Ser), L-threonine (Thr), L-tyrosine (Tyr), L-valine (Val) at $2.5 \mu \mathrm{mol} \mathrm{mL} \mathrm{m}^{-1}$ and L-cystine at $1.25 \mu \mathrm{mol} \mathrm{mL} \mathrm{m}^{-1}$, was also obtained from Sigma-Aldrich. Heptadecanoic $(\mathrm{C} 17,99.3 \%)$, and tetracosanoic $(\mathrm{C} 24,99.3 \%)$ acids, ethyl chloroformate $(\mathrm{ECF},>98 \%)$ and iso-octane $(>$ 99.5\%) were obtained from Fluka (Gillingham, Dorset). Acetonitrile (ACN), hexane, methanol, ethanol, and chloroform were of analytical-reagent or HPLC grade and were obtained from Fisher Scientific UK Limited (Loughborough, Leics.). Hydrochloric acid (0.1 M and $1 \mathrm{M})$ was volumetric analysis grade and also obtained from Fisher Scientific UK Limited. Sodium hydroxide pearls ( $>98 \%)$, purchased from Phillip Harris (Shenstone, Lichfield), were used to prepare a 1\% w/v solution in deionised water. Mylar ${ }^{\circledR}$ 
002, polyester film, $23 \mu \mathrm{m}$ thickness, was obtained from DuPont Teijin Films (UK) Limited

(Middlesbrough, UK).

\section{Preparation of calibration samples for quantification}

Equimolar amino acid calibration solutions were prepared in $0.1 \mathrm{M}$ hydrochloric acid, using the amino acid standard solution AA-S-18 and solutions prepared from individual standards, ranging between 1.25 and 200 $\mathrm{nmol} \mathrm{mL} L^{-1}$. Equimolar fatty acid solutions in hexane were prepared ranging between 5 and $1200 \mathrm{nmol} \mathrm{mL}^{-}$ 1.

Amino acid and fatty acid calibration samples were prepared, extracted, derivatised and analysed by GCMS each day. For each calibration sample, $25 \mu \mathrm{L}$ amino acid calibration solution were applied to a $2 \mathrm{~cm}$ by $2 \mathrm{~cm}$ piece of Mylar $^{\circledR} 002$ polyester film (pre-washed in each of methanol, 1\% (w/v) sodium hydroxide and acetone in turn) and allowed to dry at room temperature overnight (maximum 15 hours). Twenty-five microlitres of the corresponding fatty acid calibration solution were applied to the sample on top of the amino acids and allowed to dry (5 min). Eight microlitres nonadecanoic acid $\left(1.875 \mu \mathrm{mol} \mathrm{mL} \mathrm{m}^{-1}\right.$ in hexane, fatty acid internal standard) and $8 \mu \mathrm{L} p$-chlorophenylalanine $\left(0.3125 \mu \mathrm{mol} \mathrm{mL} \mathrm{m}^{-1}\right.$ in methanol, amino acid internal standard) were applied in turn. The samples were allowed to dry fully for 10 minutes.

Squalene calibration standards for GC-FID analysis were prepared by depositing $25 \mu \mathrm{L}$ squalene $(0-5$ $\mu \mathrm{mol} \mathrm{mL} \mathrm{m}^{-1}$ in chloroform) and $8 \mu \mathrm{L}$ nonadecanoic acid $\left(1.875 \mu \mathrm{mol} \mathrm{mL}^{-1}\right.$ in hexane $)$ on a 2 by $2 \mathrm{~cm}$ piece of Mylar $^{\circledR}$ (pre-washed as described previously) and allowed to dry.

\section{Collection of latent fingerprint samples}

Fingerprint samples were collected in accordance with the University of Lincoln ethical approval protocol (Table 1). Donors gave their informed consent and all samples and donor information were anonymised and identified using an alphanumeric code. Donors were requested not to have washed their hands in the hour preceding sampling. Donors rubbed their hands together to evenly distribute skin surface residues. Two fingerprints were deposited on a Mylar ${ }^{\circledR} 002$ strip $(10 \mathrm{~cm} \times 2 \mathrm{~cm}$, pre-washed as described above) 
from each of the left middle, left ring, right ring and right middle fingers, with as little overlap as possible (i.e. eight fingerprints deposited in total). The donor's fingers were placed onto the substrate by the researcher to control the amount of pressure applied and contact time. These fingerprint samples are referred to as the 'natural' samples.

The donor then rubbed their fingers across the forehead and nose regions of their face for approximately 10 seconds and subsequently rubbed their hands together as previously. The procedure described above was repeated to collect samples for chemical analysis. These samples are referred to as the 'groomed' samples.

Thirty minutes after collection, $8 \mu \mathrm{L} \mathrm{L}-p$-chlorophenylalanine in methanol $\left(0.3125 \mu \mathrm{mol} \mathrm{mL}^{-1}\right)$ and $8 \mu \mathrm{L}$ nonadecanoic acid in hexane $\left(1.875 \mu \mathrm{mol} \mathrm{mL}^{-1}\right)$ were applied evenly to each fingerprint sample collected for chemical analysis. Negative controls were prepared in the same way, using a 10 x $2 \mathrm{~cm}$ strip of Mylar ${ }^{\mathbb{B}}$ without the addition of fingerprints.

\section{Extraction and derivatisation of sample}

For each fingerprint and calibration sample, the Mylar ${ }^{\circledR}$ was cut smaller into $5 \mathrm{~mm}$ by $5 \mathrm{~mm}$ squares using a scalpel and glass Petri dish which were cleaned with acetone and ethanol between samples. The sample was then placed in a $4 \mathrm{~mL}$ amber glass vial with $1 \mathrm{~mL}$ of $1 \%(\mathrm{w} / \mathrm{v})$ sodium hydroxide, ethanol and pyridine, 75:40:10 v/v/v. After 1 hour on a shaker $\left(200\right.$ cycles $\left.\min ^{-1}\right), 200 \mu \mathrm{L}$ ECF:iso-octane 1:3 (v/v) were added and the sample vortexed for 30 seconds. After 1 minute, $40 \mu \mathrm{L}$ pyridine and $50 \mu \mathrm{L}$ ECF were added, vortexed for a further 30 seconds and left to stand for 2-3 minutes. The solvent was then transferred to a second $4 \mathrm{~mL}$ amber vial containing $1 \mathrm{~mL}$ chloroform with $1 \%(\mathrm{v} / \mathrm{v})$ ECF. The sample was vortexed for 30 seconds and allowed to separate. The upper aqueous layer was removed and discarded. One millilitre hydrochloric acid $(1 \mathrm{M})$ was then added and the sample vortexed for 30 seconds. The lower organic phase was transferred to a $2 \mathrm{~mL}$ amber vial and was reduced in volume to approximately $500 \mu \mathrm{L}$ under a stream of nitrogen at room temperature. The sample was transferred to a $200 \mu \mathrm{L}$ autosampler vial in aliquots and evaporated to dryness. Fifty microlitres chloroform were then added and the sample sonicated for 1 minute. Samples were not injected until at least 30 minutes after chloroform addition. 


\section{GC-MS Analysis}

Samples were analysed on a Thermo Finnigan Trace GC coupled with a Thermo Finnigan Trace MS (Mass Spec UK Ltd, Oldham, UK). One microlitre aliquots were injected onto a DB-17ms fused silica capillary column (30 m x $0.25 \mathrm{~mm}$ internal diameter (i.d.) x $0.15 \mu \mathrm{m} 50 \%$ phenylmethyl silicone film thickness, J \& W Scientific, Folsom, CA) in splitless mode. The column was held at $100{ }^{\circ} \mathrm{C}$ for 4.5 minutes, ramped to $190{ }^{\circ} \mathrm{C}$ at $10{ }^{\circ} \mathrm{C} \min ^{-1}$, then to $250{ }^{\circ} \mathrm{C}$ at a rate of $6{ }^{\circ} \mathrm{C} \mathrm{min}^{-1}$ and finally held for 10 minutes. The carrier gas was helium $\left(1 \mathrm{~mL} \mathrm{~min}^{-1}\right)$ and injector and transfer line temperatures were $250{ }^{\circ} \mathrm{C}$.

The mass spectrometer was operated in scan and selected ion monitoring (SIM) mode. All samples were analysed in SIM mode for quantification. Fingerprint and negative control samples were also analysed in scan mode for qualitative purposes. In scan mode, the spectrometer was set to monitor ions 50 to $500 \mathrm{~m} / \mathrm{z}$ with a scan time of 1 second, following a 4.5 minute solvent delay. In SIM mode, the spectrometer was set to monitor at least three characteristic ions per compound. For the positive identification of a compound, ion ratio matches for three characteristic ions (within $\pm 25 \%$ range of standard) and a relative retention time match ( \pm 3 s.d. range of standard) were required [22].

\section{GC-FID Analysis}

Squalene analysis was carried out on a Perkin Elmer Clarus 500 Gas Chromatograph with flame ionisation detection (GC-FID), using a CP Sil 5CB column (15 m x $0.25 \mathrm{~mm}$ i.d. x $0.25 \mu \mathrm{m} 100 \%$ dimethylpolysiloxane film thickness, Varian, Lake Forest, CA). A $1 \mu \mathrm{L}$ aliquot was injected in splitless mode in duplicate. The column was held at $65^{\circ} \mathrm{C}$ for 10 minutes, ramped to $125^{\circ} \mathrm{C}$ at $10{ }^{\circ} \mathrm{C} \mathrm{min}{ }^{-1}$ and held for 5 minutes. It was then ramped at $10{ }^{\circ} \mathrm{C} \min ^{-1}$ to $170{ }^{\circ} \mathrm{C}$, held for 2 minutes, ramped at $5{ }^{\circ} \mathrm{C} \min ^{-1}$ to 250 ${ }^{\circ} \mathrm{C}$ and held for a further 5 minutes. Squalene calibration standards, 'natural' and 'groomed' fingerprint samples and negative controls were injected in duplicate and analysed as described. 


\section{Statistical analysis}

Statistical analyses (t-tests and paired t-tests) were performed using SPSS 14.0, with $95 \%(P<0.05)$ confidence level to determine significant quantitative differences between donors for each compound. Principal component analysis (PCA) was carried out on the amino acid, fatty acid and squalene quantitative data using TANAGRA version 1.4.31 to identify similarities and differences between donors and sample types [23].

\section{Results and Discussion}

Latent fingerprints were collected on a non-porous substrate from a small population of donors. Amino acids and fatty acids were extracted and derivatised to form $N(O, S)$-ethoxycarbonyl amino acid ethyl esters and fatty acid ethyl esters respectively, which were identified and quantified by gas chromatography-mass spectrometry (Table 2).

Eighteen donors participated, nine males and nine females, ranging in age between 18 and 57 years old (Table 1). Four donors were vegetarian and four were smokers. A number of donors were taking medication and several were wearing cosmetic products or had used a moisturiser on the day of sample collection. Two types of fingerprints were collected from each donor. In the first instance 'natural' fingerprints were collected with no specific pre-treatment. Donors were only requested to have not washed their hands in the hour preceding sampling. For the second 'groomed' sample, donors were instructed to rub their fingers across their forehead and the region around the nose. This activity loads the fingertips with sebaceous secretions, which are abundant on the face $[24,25]$. This 'grooming' activity is often used in fingerprint studies prior to fingerprint deposition. The donor's fingers were applied to the substrate by the researcher to ensure that fingertip area sampled, time of contact and pressure were as consistent as possible between donors.

All fingerprint samples were analysed in SIM mode for identification and quantification of amino acids and fatty acids listed in Table 2. Compounds were identified on the basis of relative retention time (RRT) and ion ratio matches. 


\section{Fingerprint profiles}

Individual variation is apparent when the total ion chromatograms (TIC) are compared (Figure 1).

Qualitative (number of peaks present) and quantitative (peak size) differences can be seen between individuals and also between the 'natural' and 'groomed' samples (note the differences in ordinate scales). Target compound chromatograms (TCC) further emphasise sample variation (Figure 2). To construct the TCC, target compounds (fatty acids and amino acids listed in Table 2 in addition to squalene) were identified in the TIC and the base peak ion of the mass spectrum quantified and plotted against retention time. It should be noted that the ordinates in the TIC and the TCC are total detector response and base peak ion area respectively, and therefore the relative distribution of target compounds appears different in the two chromatograms. The TCC is simpler than its corresponding TIC allowing for more effective comparison and pattern recognition. Background compounds, which include phthalates such as diethyl-, dibutyl- and bis(2-ethylhexyl)- thought to arise from the Mylar ${ }^{\circledR}$ film (also present in the negative controls), are particularly prominent in the TIC (for example 14.78, 15.10, 17.60 and 26.39 minutes) but are not included in the TCC.

\section{Fatty acid composition}

Octanoic acid, decanoic acid, undecanoic acid, dodecanoic acid and cis-9-tetradecenoic acid gave quadratic calibration curves over the range $5-1200 \mathrm{nmol} \mathrm{mL}^{-1}$ with a correlation coefficient $\left(\mathrm{R}^{2}\right)$ exceeding 0.99 in all cases with the exception of octanoic acid (0.92) and undecanoic acid (0.97). All other fatty acids gave linear calibration curves over the same concentration range, with $\mathrm{R}^{2}$ values exceeding 0.99 in most cases and 0.97 in all. For quantification all samples were corrected using appropriate blanks. Dodecanoic acid, tetradecanoic acid, pentadecanoic acid, hexadecanoic acid, octadecanoic acid and cis-9-octadecenoic acid were positively identified in all samples and in most cases exceeded the limit of quantification (200 - 394 ng deposited, mean blank +10 standard deviations, [26]). Tridecanoic acid, cis-9-hexadecenoic acid, heptadecanoic acid, cis-9,12-octadecadienoic acid, eicosanoic acid and tetracosanoic acid were positively identified and above the limit of detection (96 - 464 ng deposited, mean blank +3 standard deviations [26]) in some samples but not above the limit of quantification in most cases (137 - 570 ng deposited). The 
remaining fatty acids, octanoic, decanoic, undecanoic and cis-9-tetradecenoic acids, were not positively identified in any fingerprint samples.

Total fatty acid deposited ranged from 0.16 to $3.37 \mu \mathrm{g}$ per fingerprint, for those positively identified. There is wide variation in the relative amounts of each fatty acid found in the latent fingerprint samples (Figure 3). Hexadecanoic acid was the most abundant in all samples followed by octadecanoic acid and cis-9octadecenoic acid, consistent with previously published data $[13,15,27]$. Quantitative differences were found between the 'natural' and 'groomed' fingerprint samples (Figure 3a). Significantly $(P<0.05)$ greater amounts of fatty acids were deposited in the latter, consistent with the act of 'grooming' and touching sebum-rich areas of the face immediately prior to depositing the fingerprints. In Figure $3 b$ the amount of each fatty acid is normalised to hexadecanoic acid in the sample. The qualitative differences between the 'natural' and 'groomed' sample types were less marked.

Variation is evident in the distribution of individual fatty acids between donors (Figure 3). This may be due to donor-dependent factors such as diet or cosmetic use. Comparison of the differences between the two sample types for individual donors reveals further variation. The increase in individual fatty acids after the act of grooming varied from 1\% ('natural' sample $29.93 \mathrm{ng}$ tetradecanoic acid per fingerprint, 'groomed' sample $30.23 \mathrm{ng}$ ) to over $2000 \%$ (28.54 to $650.92 \mathrm{ng}$ cis-9-octadecenoic acid). The decrease in material deposited varied from $12 \%$ (43.39 to $38.26 \mathrm{ng}$ octadecanoic acid) to almost complete absence. The percentage increase or decrease for any given donor was not consistent for all fatty acids indicating differential distribution on both the fingertips and the face. Overall, the increase in total fatty acid after grooming was 22-2000\%, which clearly demonstrates the significant impact the sampling protocol has on the chemical composition of the latent fingerprints deposited.

Only one donor, Donor 18, deposited less material overall in their 'groomed' sample (4.5\%) compared to their 'natural' sample. Whilst there was an overall decline in fatty acid deposited, there was an increase seen for hexadecanoic (5\%), cis-9-octadecenoic (8\%), and octadecanoic acids (17\%). Donor 18 was an 18 year-old female donor, who reported taking the antibiotic erythromycin. Antibiotics affect bacterial lipase 
activity responsible for the hydrolysis of triglycerides in sebum secreted onto the skin and therefore would reduce the levels of selected free fatty acids available to be picked up during 'grooming' [28]. The sample population in this study is, however, limited.

The relative distribution of tetradecanoic, octadecanoic and cis-9-octadecenoic acids (normalised using hexadecanoic acid) in the 'natural' and 'groomed' samples were consistent with the studies of Mong et al. [13] and Archer et al. [15], with the exception of octadecanoic acid which was present in greater relative amounts in the latter study. Mong et al. and Archer et al. collected fingerprints on filter paper and their sampling protocols included the donors rubbing their fingertips across the forehead prior to depositing fingerprints. Consequently absolute quantitative comparisons are difficult between the studies.

\section{Amino acid composition}

All amino acids gave linear calibration curves over the concentration range $1.25-200 \mathrm{nmol} \mathrm{mL}^{-1}$ with $\mathrm{R}^{2}$ values exceeding 0.99 in most cases and 0.95 in all. Arginine, cystine, and histidine were not detected in any calibration standards or fingerprint samples. Hydroxyproline, methionine and tryptophan were not positively identified in any fingerprint samples, consistent with previous studies where they were detected at very low levels or not at all $[18,19,20]$. Lysine, ornithine and tyrosine were detected and positively identified in most fingerprint samples. However, they behaved inconsistently in the calibration standards and therefore accurate quantification was not possible in this study. These amino acids will not be discussed further.

Glycine, valine, leucine, serine, proline, asparagine, aspartic acid, glutamic acid, phenylalanine and cysteine were positively identified in all fingerprint samples with a few exceptions. Alanine was at least partially identified (one ion ratio or more) in most samples. Qualitative ion peaks $(70,144,189 \mathrm{~m} / \mathrm{z}$ ) were often difficult to integrate or absent. No ion ratio matches were found for isoleucine in approximately half of the samples. Threonine was positively identified in most samples but was not found in levels greater than the negative controls in any samples except two. In most cases all samples were found to be above the limit of quantification for most amino acids (15.5 - 28.1 ng deposited). 
Serine was the most abundant amino acid followed by glycine, alanine and aspartic acid. The relative abundance of amino acids was in general consistent with previously published studies [18,19,20]. Wide variation is observed between the donors with total amino acid deposited ranging from 20.7 to $345.1 \mathrm{ng}$ per fingerprint. There is no correlation between total amount of fatty acid and total amino acid deposited in the 'natural' fingerprint samples (Figure 4a) as expected since they are predominantly from different sources, namely sebaceous secretions and eccrine sweat respectively. There is also no correlation for the 'groomed' fingerprint samples (Figure 4b).

The significant quantitative differences between the 'natural' and 'groomed' fingerprint samples seen for the fatty acids were not observed in the case of the amino acids $(P>0.05$, Figure 5a). The data indicate that the predominantly lipid material, picked up on the fingertips during the 'grooming' procedure, did not inhibit the extraction of amino acids from the fingerprint using this experimental procedure. If this had been the case, the amino acid levels in the 'groomed' samples would have been consistently lower. For over half of the donors $(n=10)$ there was a decline in the total amount of amino acid deposited in the 'groomed' sample compared to the 'natural' sample and an increase for the remaining donors. A consistent increase (or decrease) was not always seen, however, for all individual amino acids for a given donor. For some donors, selected amino acids were only found in either the 'groomed' or the 'natural' sample. In the sampling protocol employed, the 'natural' sample was collected first and there was a minimal time delay before the collection of the 'groomed' sample (typically 10 minutes, exact time dependent on length of time taken to deposit all fingerprint samples and conduct 'grooming' procedure). It is expected that the amino acids present on the fingertips are depleted to some extent in the first fingerprint sample and are not replenished by the 'grooming' procedure and thus lower amounts of amino acids are expected in the 'groomed' samples. The interactions between the eccrine and sebaceous components on the fingertip and with the non-porous substrate are not clearly understood. It may be that the amino acids were not fully depleted in the first sample. Amino acids may be picked up on the fingertips from the forehead during grooming [29,30]. Qualitatively, the 'natural' and 'groomed' samples were comparable, with the 
exceptions of the relative amounts of alanine and glycine, which appear to be more abundant in the former

(Figure 5b). The differences in relative abundance were significant in the case of glycine $(P<0.05)$.

\section{Squalene}

Squalene was identified in the fingerprint samples by mass spectral analysis and consequently the fingerprint samples were re-analysed by GC-FID for quantification. The squalene content of the fingerprint samples is summarised in Figure 6. Significantly greater amounts of squalene were deposited in the 'groomed' sample compared to the 'natural' sample $(P<0.001)$. The amount of squalene increased by between 1.5 to 68.4 times, as expected since squalene is derived from sebaceous secretions [24,25]. As seen for the fatty acids, quantitative variation was evident between donors. For example, the 'natural' fingerprint samples of Donor 4 and Donor 17 contained comparable amounts of squalene, 124.25 and 124.24 ng per fingerprint respectively. However, their 'groomed' samples were very different with that of Donor 4 (1282.00 ng) containing more than double Donor 17's sample (623.15 ng). Squalene was not found in the 'natural' samples of Donor 9 and following 'grooming' $283.01 \mathrm{ng}$ per fingerprint were deposited.

The relative squalene content (normalised to hexadecanoic acid) of the samples in this study were again compared with those of Mong et al. [13] and Archer et al. [15]. The 'groomed' samples were comparable to those of Mong et al., whilst the 'natural' samples were lower and comparable to the Archer et al. samples.

\section{Individual variation and personal traits}

Characteristic compounds may be found in a latent fingerprint which could potentially be exploited to provide donor information such as age or a personal habit $[4,5,31]$. The individual amino acid and fatty acid composition data were studied to determine whether any quantitative variation arose as a consequence of a particular donor attribute, namely gender, diet, age and smoking. 
Gender differences in the amino acid content of sweat have been reported previously [30]. Individual mean amino acid levels were higher in females $(n=9)$ compared to males $(n=9)$ but only in the case of asparagine in the 'natural' samples was this difference significant $(P<0.05)$. Conversely, for most fatty acids the mean amount per fingerprint was higher in the male samples compared to the female samples, although this was not significant $(P>0.05)$. Penn et al. [32] reported that pentadecanoic, hexadecanoic and heptadecanoic acids in axillary sweat were characteristic of male subjects. Pentadecanoic and hexadecanoic acids were found in this study to be in greater abundance in the male 'groomed' samples compared to the female 'groomed' samples, although this was not significant $(P>0.05)$. Quantitative differences between the 'ungroomed' samples were smaller. It should be noted that individual quantitative differences may arise as a consequence of differences in fingerprint surface area.

Amino acid content was found to differ between the vegetarian donors $(n=4)$ and those with an omnivorous diet $(\mathrm{n}=14)$. In the 'natural' samples most amino acids were comparable. The mean amount of alanine, glycine and serine was higher for the vegetarian donors although not significantly $(P>0.1)$. These differences were not evident in the 'groomed' samples. For the fatty acids, composition was comparable for the 'natural' samples. Pentadecanoic acid, however, differed significantly between the omnivores and vegetarians $(P<0.05)$. Comparing the 'groomed' samples, mean amounts were higher in the omnivore samples compared to the vegetarians. Striking differences were evident for hexadecanoic and cis-9-octadecenoic acids, although a significant difference was found only for the latter $(P<0.01)$.

No significant differences were found between the non-smokers $(\mathrm{n}=14)$ and the smokers $(\mathrm{n}=4)$ for any amino acid or fatty acid. The donors were arbitrarily divided into '20 years and under' $(\mathrm{n}=12)$ and '21 years and over' $(\mathrm{n}=6)$ groups. Significant differences $(P<0.05)$ between the two groups were found for alanine, glycine and valine in the 'natural' samples. In the case of the fatty acids, a significant difference was seen only for dodecanoic acid in the 'natural' samples $(P<0.05)$.

Based on this limited quantitative data, no compound has been identified as a marker for a particular donor trait. This was as expected since these compounds are generic and commonly found in sweat and 
sebaceous secretions. It should be noted that the variation seen will not be mutually exclusive and only due to one particular donor trait, but will be a consequence of a combination of contributory factors.

Principle component analysis was performed of the entire target compound quantitative data in order to determine whether it is possible to differentiate different sample types using all compounds. Figure 7 shows the principle component scores plot of PCA1 and PCA2, which account for $82 \%$ of the variance observed in the population. The scores plot illustrates that the 'natural' and 'groomed' samples may be differentiated although there is some overlap. The differences between the male and female samples are less marked. As expected the fatty acid and squalene variables showed close correlation and were negatively correlated to the amino acids along PCA2. This would suggest that PCA2 gives discrimination between 'natural' and 'groomed' samples of which there is some evidence in Figure 7 and is consistent with the observations in Figures 3 and 5. There was no discrimination between samples on the basis of the other donor attributes namely diet, age and smoking.

\section{Conclusions}

This study further develops our current understanding of latent fingerprint composition. The amino acid, fatty acid and squalene composition of latent fingerprints was found to vary quantitatively between individual donors in this study. Whilst many of the compounds studied were found to be common to all samples, the relative amounts were found to differ. The impact of the sampling protocol on the quantitative analysis of fingerprints has also been clearly demonstrated. The act of 'grooming' prior to depositing latent fingerprints had a significant effect quantitatively, and to a lesser extent qualitatively, on the fatty acid composition of the samples. Although the same marked effect was not seen for all amino acids, 'grooming' did affect some. Principal component analysis allowed the differentiation of the 'natural' and 'groomed' fingerprint samples. There was no such differentiation found on the basis of gender, diet, age or smoking. 
The need to consider the sampling protocol when conducting and evaluating latent fingerprint studies has been highlighted. Analyses here indicate that the use of 'groomed' or 'enhanced' fingerprints could seriously compromise the validity of fingerprint reagent assessments. This would be particularly so with regard to reagents for sebaceous components where, on average, fatty acid content could be over represented by a factor of four. Squalene could be over represented by a factor of sixteen and total sebaceous mass over represented by a factor of six. Use of 'natural' fingerprints is therefore recommended for all trials of reagents. 


\section{References}

[1] V. Bowman (Ed.), Manual of Fingerprint Development Techniques, $2^{\text {nd }}$ rev. ed., White Crescent Press Ltd., Luton, U.K., 2004.

[2] J. Almog, Y. Cohen, M. Azoury, T.-R. Hahn, Genipin - A novel fingerprint reagent with colorimetrc and fluorogenic activity, J. Forensic Sci. 49 (2004) 255-257.

[3] J. Almog, A. Klein, I. Davidi, Y. Cohen, M. Azoury, M. Levin-Elad, Dual fingerprint reagents with enhanced sensitivity: 5-methoxy- and 5-methylthioninhydrin, J. Forensic Sci. 53 (2008) 364-368.

[4] R. Leggett, E.E. Lee-Smith, S.M. Jickells, D.A. Russell, "Intelligent" fingerprinting: Simultaneous identification of drug metabolites and individuals by using antibody-functionalized nanoparticles, Angew. Chem. Int. Ed. 46 (2007) 4100-4103.

[5] P. Hazarika, S.M. Jickells, K. Wolff, D.A. Russell, Imaging of latent fingerprints through the detection of drugs and metabolites, Angew. Chem. Int. Ed. 47 (2008) 10167-10170.

[6] V. Drapel, A. Becue, C. Champod, P. Margot, Identification of promising antigenic components in latent fingerprint residues, Forensic Sci. Int. 184 (2009) 47-53.

[7] C.G. Worley, S.S. Wiltshire, T.C. Miller, G.J. Havrilla, V. Majidi, Detection of visible and latent fingerprints using micro-x-ray fluorescence elemental imaging, J. Forensic Sci. 51 (2006) 57-63.

[8] C. Ricci, P. Phiriyavityopas, N. Curum, K.L.A. Chan, S. Jickells, S.G. Kazarian, Chemical imaging of latent fingerprint residues, Appl. Spectrosc. 61 (2007) 514-522.

[9] A. Hemmila, J. McGill, D. Ritter, Fourier transform infrared reflectance spectra of latent fingerprints: a biometric gauge for the age of an individual, J. Forensic Sci. 53 (2008) 369-376.

[10] M.I. Szynkowska, K. Czerski, J. Rogowski, T. Paryjczak, A. Parczewski, ToF-SIMS application in the visualization and analysis of fingerprints after contact with amphetamine drugs, Forensic Sci. Int. 184 (2009) e24-e26.

[11] R.S. Ramotowski, Composition of latent print residue, in: H.C. Lee, R.E. Gaensslen (Eds.), Advances in Fingerprint Technology, 2nd ed., CRC Press, Boca Raton, Florida, 2001, pp. 63-104.

[12] M.V. Buchanan, K. Asano, A. Bohanan, Chemical characterization of fingerprints from adults and children, SPIE Proceedings: Forensic Evidence Analysis \& Crime Scene Investigation 2941 (1997) 89-95. 
[13] G. Mong, C.E. Petersen, T.R.W. Clauss, Advanced fingerprint analysis project. Final report fingerprint constituents, Pacific Northwest National Laboratory Report, 1999.

[14] K.G. Asano, C.K. Bayne, K.M. Horsman, M.V. Buchanan, Chemical composition of fingerprints for gender determination, J. Forensic Sci. 47 (2002) 805-807.

[15] N.E. Archer, Y. Charles, J.A. Elliott, S. Jickells, Changes in the lipid composition of latent fingerprint residue with time after deposition on a surface, Forensic Sci. Int. 154 (2005) 224-239.

[16] K.A. Mountfort, H. Bronstein, N. Archer, S.M. Jickells, Identification of oxidation products of squalene in solution and in latent fingerprints by ESI-MS and LC/ACPI-MS, Anal. Chem. 79 (2007) 26502657.

[17] D.B. Hansen, M.M. Joullié, The development of novel ninhydrin analogues, Chem. Soc. Rev. 34 (2005) 408-417.

[18] P.B. Hamilton, Amino-acids on hands, Nature 205 (1965) 284-285.

[19] B. Hadorn, F. Hanimann, P. Anders, H.C. Curtius, Free amino-acids in human sweat from different parts of the body, Nature 215 (1967) 416-417.

[20] J. Oro, H.B. Skewes, Free amino-acids on human fingers: The question of contamination in microanalysis, Nature 207 (1965) 1042-1045.

[21] F. Cuthbertson, The chemistry of fingerprints, AWRE Report No. 013/69, Atomic Energy Authority, U.K., 1969.

[22] R.S. Croxton, M.G. Baron, D. Butler, T. Kent, V.G. Sears, Development of a GC-MS method for the simultaneous analysis of latent fingerprint components, J. Forensic Sci. 51 (2006) 1329-1333.

[23] R. Rakotomalala, TANAGRA: un logiciel gratuity pour l'enseignement et la recherché, Actes de EGC'2005, RNTI-E-3 2 (2005) 697-702.

[24] R.S. Greene, D.T. Downing, P.E. Pochi, J.S. Strauss, Anatomical variation in the amount and composition of human skin surface lipid, J. Invest. Dermatol. 54 (1970) 240-247.

[25] D.T. Downing, J.S. Strauss, Synthesis and composition of surface lipids of human skin, J. Invest. Derm. 62 (1974) 228-244.

[26] Eurachem, The fitness for purpose of analytical methods. A laboratory guide to method validation and related topics, LGC, Teddington, Middlesex, 1998. 
[27] J.D. Wilson, D.J. Darke, The results of analyses of the mixtures of fatty acids found on the skin Part I Commentary, Part II Tables and Figures, Harwell Ind Report, AERE rep G1154, U.K., 1978.

[28] R.R.. Marples, D.T. Downing, A.M. Kligman, Control of free fatty acids in human surface lipids by Corynebacterium Acnes, J. Invest. Dermatol. 56 (1971) 127-131.

[29] N. Liappis, H. Hungerland, Quantitative study of free amino acids in human eccrine sweat during normal conditions and exercise, Am. J. Clin. Nutr. 25 (1972) 661-663.

[30] N. Liappis, A. Jäkel, Über die ausscheidung der freien aminosäuren im menschlichen ekkrinen schweiß, Arch. Dermatol. Res. 254 (1975) 185-203.

[31] S. Haze, Y. Gozu, S. Nakamura, Y. Kohno, K. Sawano, H. Ohta, K. Yamazaki, K. 2-Nonenal newly found in human body odor tends to increase with aging, J. Invest. Dermatol. 116 (2001) 520-524.

[32] D.J. Penn, E. Oberzaucher, K. Grammer, G. Fischer, H.A. Soini, D. Wiesler, M.V. Novotny, S.J.

Dixon, Y. Xu, R.G. Brereton, Individual and gender fingerprints in human body odour, J. R. Soc. Interface 4 (2007) 331-340. 
Table 1: Summary of donor information

Table 2: Amino acids and fatty acids detected in latent fingerprint samples by gas chromatographymass spectrometry and their SIM programme parameters

Figure 1: Total ion chromatograms (TIC) of (i) 'natural' and (ii) 'groomed' latent fingerprint samples

(a) Donor 2, 57 year old male, (b) Donor 13, 18 year old female, (c) Donor 18, 18 year old female. Numbers refer to Table 2, with the exception of 21 which refers to squalene.

Figure 2: Target compound chromatograms (TCC) of (i) 'natural' and (ii) 'groomed' latent fingerprint samples

(a) Donor 2, 57 year old male, (b) Donor 13, 18 year old female, (c) Donor 18, 18 year old female. $*=$ chlorophenylalanine (amino acid internal standard); $* *=$ nonadecanoic acid (fatty acid internal standard)

Figure 3: Fatty acid content of fingerprint samples

Amount in a single fingerprint (a) in nanograms and (b) relative to C16 (hexadecanoic acid). 'Groomed' samples of Donor 2 (1472 ng, extreme value) and Donor 11 (1116.47 ng, outlier value) have been omitted from $\mathrm{C} 16$ dataset in (a). $\star$ indicates an extreme value (more than 3 times interquartile range from upper edge of box) and $\bigcirc$ indicates an outlier value (between 1.5 and 3 times interquartile range from upper edge of box). Unshaded boxes = 'natural' sample and shaded boxes $=$ 'groomed' sample.

Figure 4: Comparison of total amino acid and fatty acid content of a single (a) 'natural' and (b) 'groomed' fingerprint

Donor 12 'natural' sample has been omitted from (a) due to extreme outlier value for cysteine.

Figure 5: Amino acid content of fingerprint samples

Amount in a single fingerprint (a) in nanograms and (b) relative to serine. Donor 12 'natural' sample (594.11 ng, extreme value) has been omitted from cysteine dataset. See Figure 3 for key for $\star$ and $\bigcirc$. Unshaded boxes $=$ 'natural' sample and shaded boxes $=$ 'groomed' sample.

Figure 6: Squalene content of fingerprint samples Amount in a single fingerprint (a) in nanograms and (b) relative to $\mathrm{C} 16$ (hexadecanoic acid). See Figure 3 for key for $\star$ and $O$. Unshaded boxes $=$ 'natural' sample and shaded boxes = 'groomed' sample.

Figure 7: Principal component analysis of latent fingerprint target compounds, PCA2 versus PCA1 $\bullet$ = male 'natural'; $\triangle$ = female 'natural'; $\square=$ male 'groomed'; + = female 'groomed' 
Forensic Sci. Int., 2010, Vol. 199, 93-102

Table 1: Summary of donor information

\begin{tabular}{|c|c|c|c|c|c|c|c|c|c|c|c|c|c|}
\hline Sex & $\mathbf{N}$ & Age & & Ethnicity & & Smo & & Med & tion & $\begin{array}{l}\text { Cos } \\
\text { Cre }\end{array}$ & & Diet & \\
\hline Male & 9 & $18-29$ & 13 & Caucasian & 18 & Yes & 4 & Yes & 7 & Yes & 11 & Omnivore & 14 \\
\hline Female & 9 & $30-60$ & 5 & & & No & 14 & No & 11 & No & 7 & Vegetarian & 4 \\
\hline
\end{tabular}

Table 2: Amino acids and fatty acids detected in latent fingerprint samples by gas chromatography-mass spectrometry and their SIM programme parameters

\begin{tabular}{clll}
\hline Compound & $\begin{array}{l}\text { Retention time } \\
(\mathbf{m i n})\end{array}$ & $\begin{array}{l}\text { Characteristic ions } \\
\text { (m/z [relative abundance(\%)]) }\end{array}$ \\
\hline $1^{\text {a }}$ & Alanine & 9.44 & $70[7.4], 116^{\mathrm{b}}[100], 144[1.7], 189[0.4]$ \\
2 & Glycine & 9.92 & $74[15], \underline{102}[100], 130[5.6], 175[4.2]$ \\
3 & Valine & 11.02 & $72[34], 116[38], 144[100]$, \\
4 & Leucine & 11.96 & $86[8.3], 102[39], 129[4.2], \underline{158}[100], 231$ \\
5 & Isoleucine & 12.19 & {$[0.3]$} \\
6 & Dodecanoic acid & 12.36 & $102[50], 129[11], 158[100]$ \\
7 & Serine & 13.18 & $101[100], 157[25], 228[4.5]$ \\
8 & Proline & 13.49 & $60[76], 86[35], 132[100]$ \\
9 & Asparagine & 13.79 & $114[10], 142[100], 170[0.8], 215[4.6]$ \\
10 & Tetradecanoic acid & 14.67 & $69[100], \underline{141}[71], 174[3.8]$ \\
11 & Aspartic acid & 15.04 & $101[100], 157[32], 256[6.1]$ \\
12 & Pentadecanoic acid & 16.01 & $116[28], 142[25], 188[100]$ \\
13 & Glutamic acid & 16.57 & $101[100], 157[27], 270[6.2]$ \\
14 & Hexadecanoic acid & 17.04 & $\underline{128}[100], 156[83], 202[75]$ \\
15 & Phenylalanine & 17.88 & $101[100], 157[28], 284[7.2]$ \\
16 & Cysteine & 18.49 & $102[73], 176[100], 192[46]$ \\
17 & Octadecanoic acid & 19.50 & $74[93], 102[100], \underline{220}[91]$ \\
18 & cis-9-Octadecenoic acid & 19.51 & $101[100], 157[26], 312[7.4], 313[1.8]$ \\
19 & p-Chlorophenylalanine & 20.36 & $180[100], 222[97], 235[15], 264[94]$ \\
20 & Nonadecanoic acid & 20.74 & $102[100], 125[70], \underline{210}[91]$ \\
\hline
\end{tabular}

${ }^{\mathrm{a}}$ Number corresponds to elution order in chromatograms in Figure 6.

${ }^{\mathrm{b}}$ Quantification ion underlined 
Figure 1
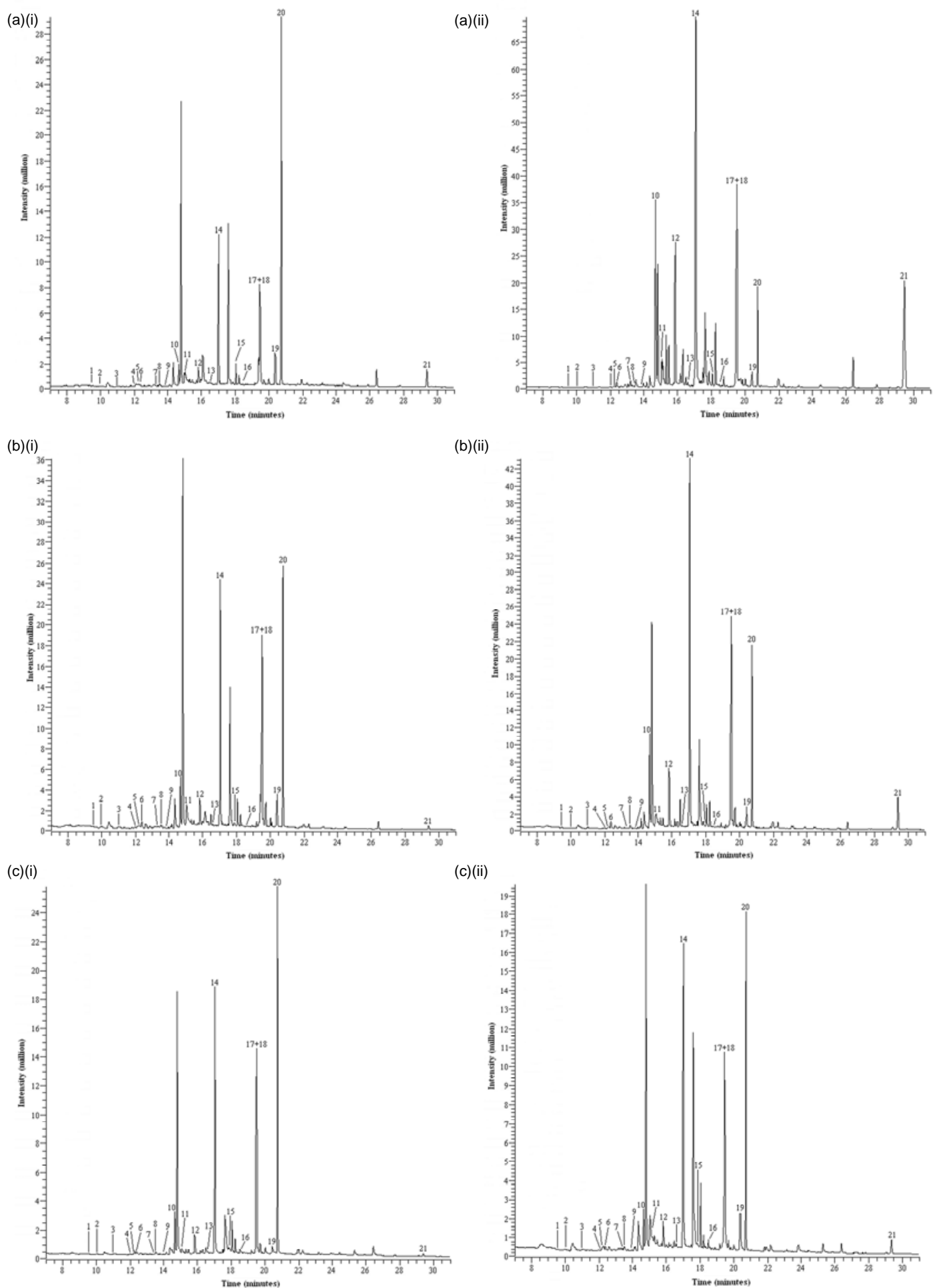


\section{Figure 2}

(a)(i)

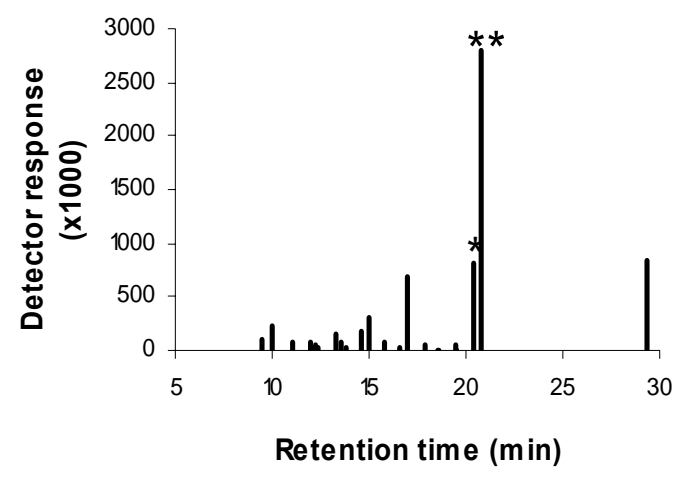

(b)(i)

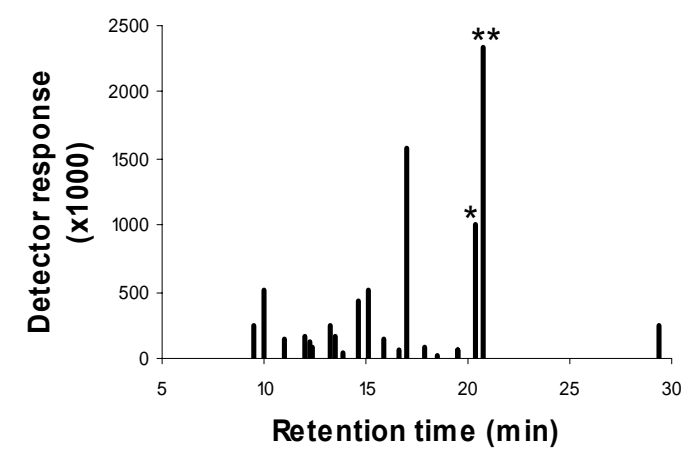

(c)(i)

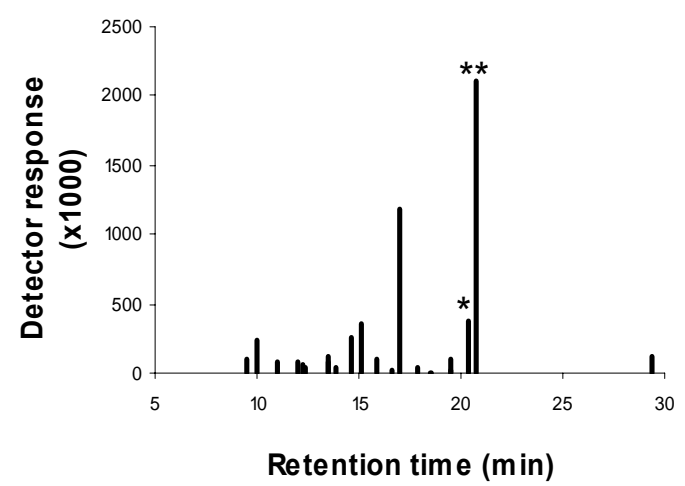

(a)(ii)

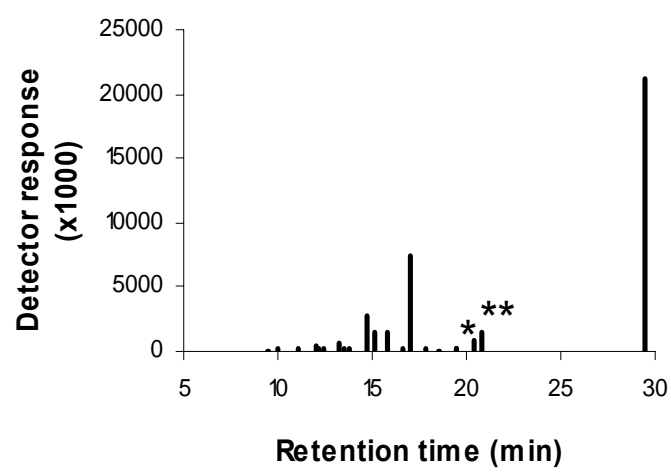

(b)(ii)

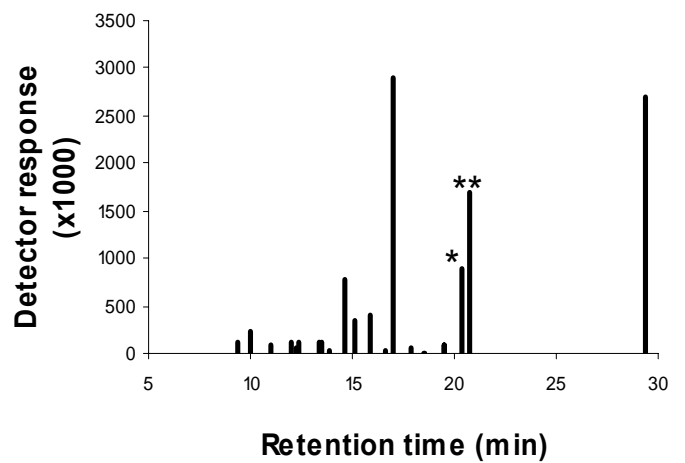

(c)(ii)

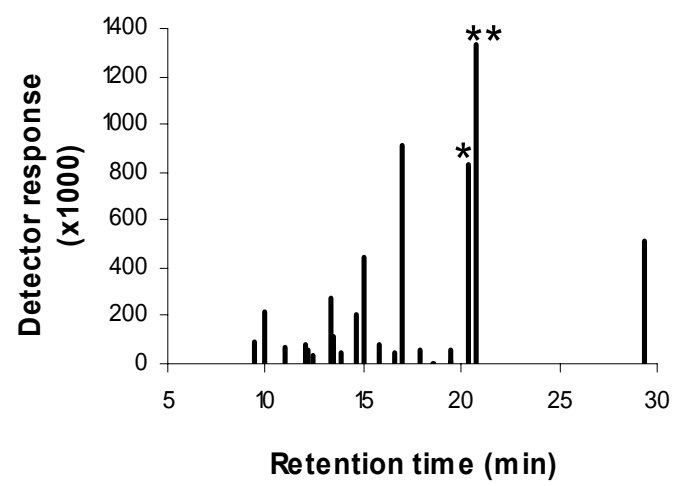


Figure 3
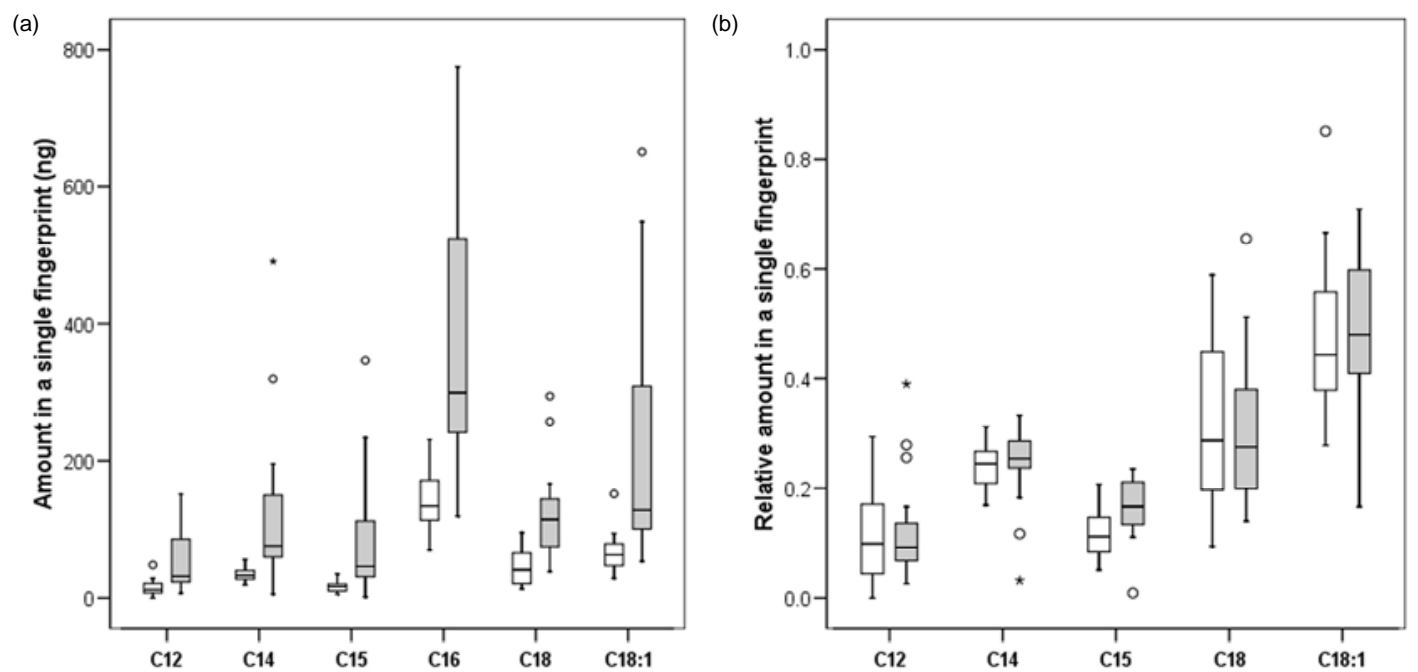
Figure 4

(a)

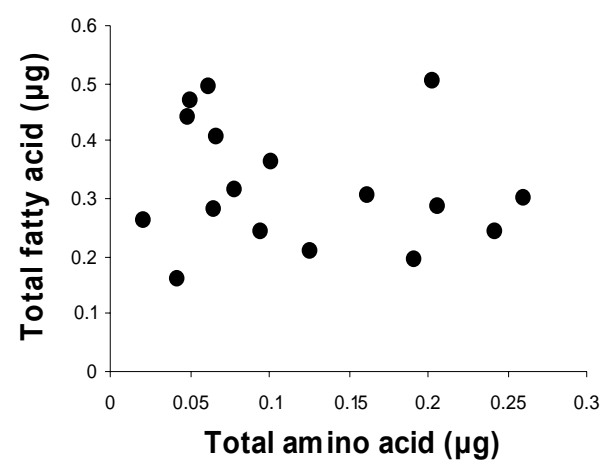

(b)

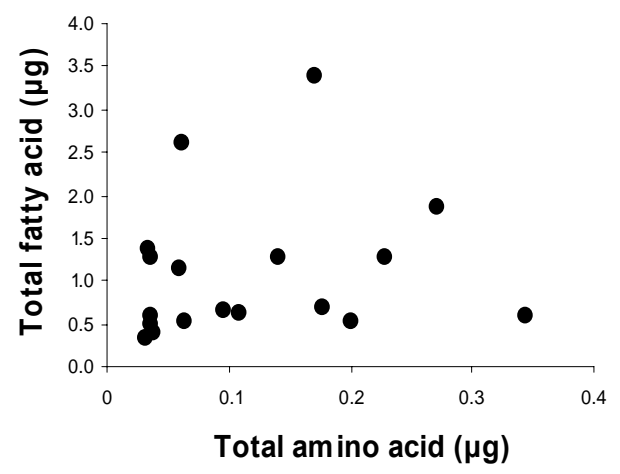


Figure 5
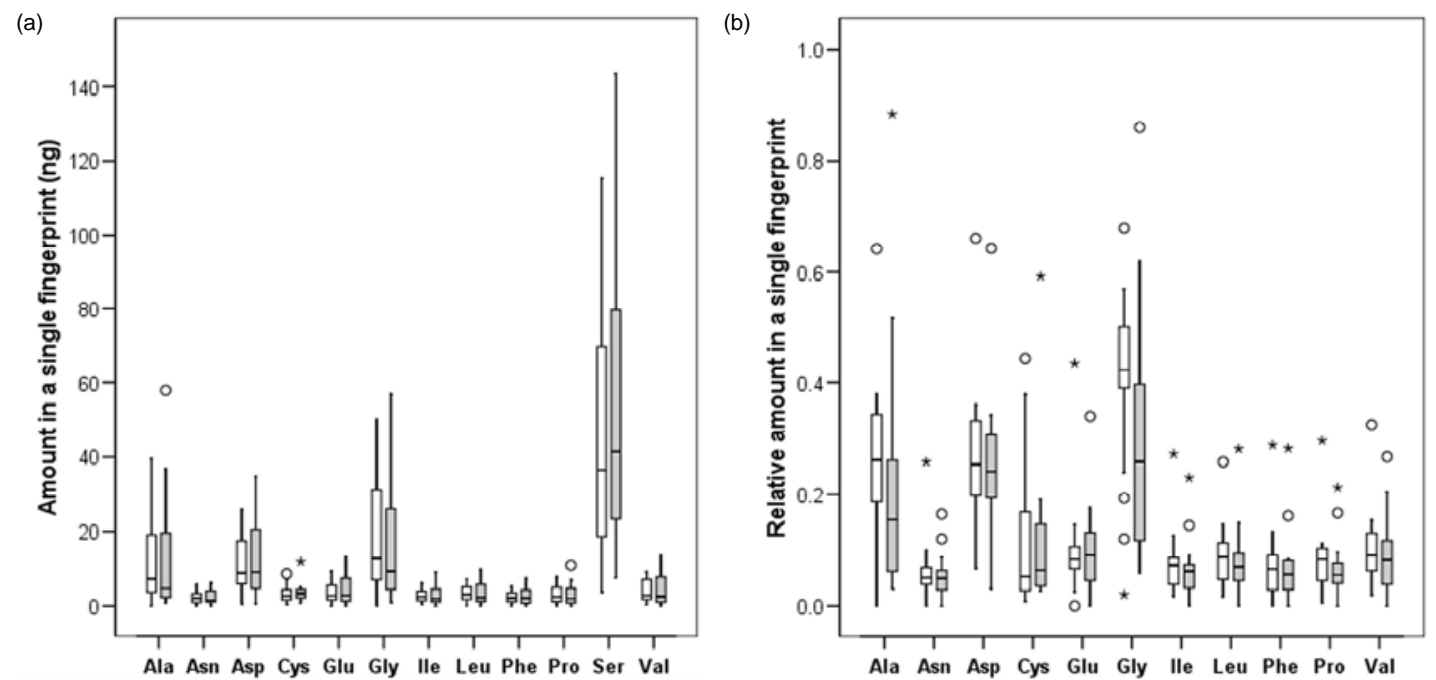
Figure 6
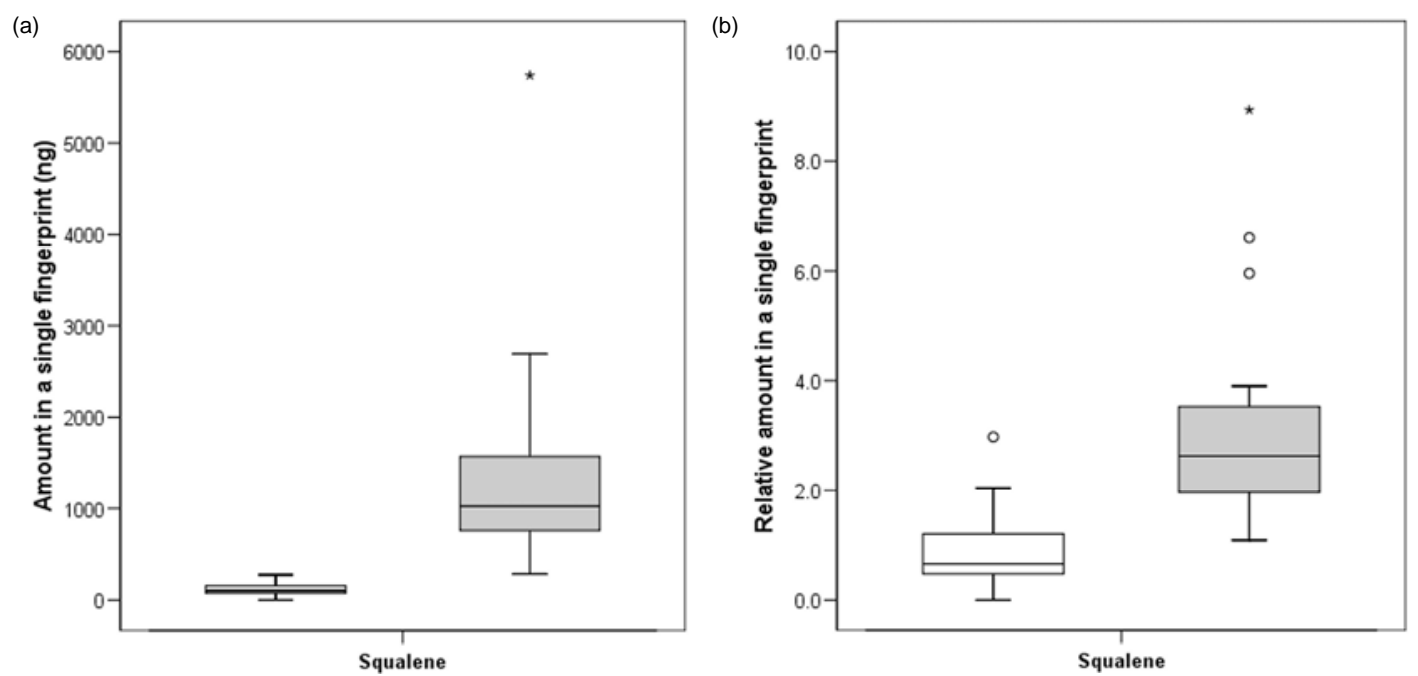
Figure 7

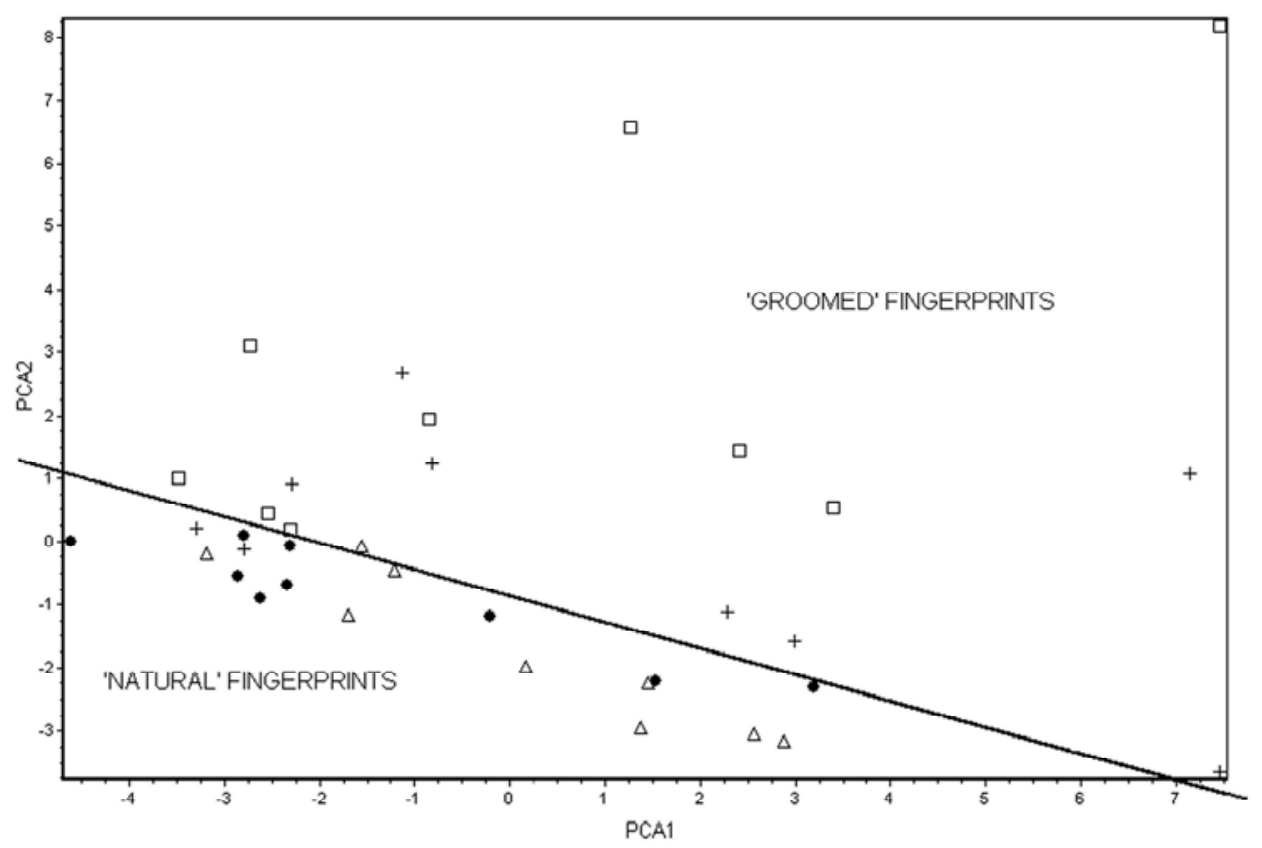

